\title{
Defense Strategy of Aircraft Confronted with IR Guided Missile
}

\author{
Hesong Huang, Zhongxiang Tong, Taorui Li, Lintong Jia, and Shenbo Li \\ Aeronautics and Astronautics Engineering College, Air Force Engineering University, No. 1 Baling Road, Baqiao District, \\ Xian 710038, China \\ Correspondence should be addressed to Hesong Huang; huanghesonghhs@126.com
}

Received 18 October 2016; Revised 4 December 2016; Accepted 7 December 2016; Published 9 January 2017

Academic Editor: Vyacheslav V. Kalashnikov

Copyright (c) 2017 Hesong Huang et al. This is an open access article distributed under the Creative Commons Attribution License, which permits unrestricted use, distribution, and reproduction in any medium, provided the original work is properly cited.

\begin{abstract}
Surface-type infrared (IR) decoy can simulate the IR characteristics of the target aircraft, which is one of the most effective equipment to confront IR guided missile. In the air combat, the IR guided missile poses a serious threat to the aircraft when it comes from the front of target aircraft. In this paper, firstly, the model of aircraft and surface-type IR decoy is established. To ensure their authenticity, the aircraft maneuver and radiation models based on real data of flight and exhaust system radiation in the state of different heights and different speeds are established. Secondly, the most effective avoidance maneuver is simulated when the missile comes from the front of the target aircraft. Lastly, combining maneuver with decoys, the best defense strategy is analysed when the missile comes from the front of aircraft. The result of simulation, which is authentic, is propitious to avoid the missile and improve the survivability of aircraft.
\end{abstract}

\section{Introduction}

With the development of IR technology, IR guided missile gradually replaces the status of radar guided missile and becomes the main weapon in the air combat. In the war area for 20 years, about $90 \%$ of aircrafts were damaged by the IR guided missile [1]. So studying the IR defense strategy is becoming necessary. In recent years, many researches have been carried out about air combat especially the optimal flight paths in air combat [2] and multiple aircrafts cooperative air combat [3], but few for IR defense strategy. IR defense is the key to IR antagonism. The aircraft can get away from missile and enemy aircraft and even counter them by performing reasonable and effective means of defense. Particularly when the missile is coming from frontage, it is the opposite moving between the aircraft and missile that makes pilot nervous, what makes the aircraft being shot down before the pilot have time to react. All in all, it is important to design reasonable and effective means of defense, which can improve aircraft susceptibility and lengthen pilots' life.

Arthur establishes the model of decoy and vertical-S maneuver and obtains the best defensive strategy that the aircraft deploys decoys and performs vertical- $S$ maneuver simultaneously [4]. But the antijamming performance of missile which tracks the center of energy is very weak in the paper. This missile has been replaced by a new generation of missile in modern air combat, so the defense strategy has not more instructions to the modern air combat. Fumiaki Imado studies defense strategy of barrel roll maneuvers against a proportional navigation guidance missile and draws the conclusion that a high- $g$ barrel roll maneuver generally produces a larger miss distance than a split- $S$ maneuver [5]. But most of the research is about avoidance maneuver, which does not analyse the interference effect of decoys. Xinhui et al. establish the mathematic model of barrel roll maneuvering and study the effect of fighter barrel roll maneuvering terminal evasion [6]. Finally, it is obtained that the miss distance becomes bigger firstly and then smaller as the change of barrel roll angle rate. But it does not contrast other maneuvers and the conclusions are not accurate.

In this paper, the best avoidance maneuver is obtained by contrasting horizontal- $S$ maneuver to barrel roll maneuver. And then according to the antijamming performance of missile and applying IR decoy into avoidance maneuver, comparing with different defense strategies, the best defense strategy is obtained which is propitious to avoid the missiles and improve the survivability of aircraft.

The main construction of the paper is that, firstly, the simulation model containing aircraft and surface-type IR decoys is established. Secondly, put the measured data to 
good use, which ensure the reliability of model. Finally, the best defensive strategy is acquired with the incoming IR guided missiles in front of the aircraft by simulation and analysis, considering the interference of maneuvers and surface-type IR decoys comprehensively.

\section{Analysis of Defensive Strategy of Aircraft Confront IR Guided Missile}

When IR guided missile is flying opposite to the aircraft, it is infeasible to avoid missile in the method of consuming missile energy by reciprocating maneuver. To avoid missile, the following points are taken into consideration.

2.1. Weaken Radiation of Aircraft Skin. The skin radiation from the front of aircraft is lowest [7]. As distance becomes a little longer, the IR radiation will not reach the threshold of the IR detector in the seeker [8]. Thus, in the process of headon attack, the missile cannot capture target aircraft. Finally, the hit ratio will drop sharply. It is obviously supposed to keep the head of the aircraft pointing to the missile all the time to avoid the head-on missile.

Supposing that the aircraft points to the coming missile, the pilot does not need to adjust. While the aircraft does not point to the coming missile in most cases, the pilot needs to adjust the aircraft to point to the coming missile constantly. However, as the missile-target distance becomes shorter, the seekers will capture enough radiation of the aircraft skin to hit it at last. It is unrealistic only by weakening radiation of aircraft skin to avoid missile, which must be combined with decoys. This paper will not do much about it.

\subsection{Perform the Maximum Overload Evadable Maneuver.} In process of attack, the speed of the missile is more than twice faster than aircraft. When the missile is close to the aircraft, with the limit of the missile self-overload, the turn radius will be very large and the turn rate may not be enough large to track the aircraft [9]. There is a great chance that the aircraft will avoid missile attack while performing the maximum overload evadable maneuver, especially snake maneuver and barrel roll maneuver [10]. Suppose that the maximum overload of the missile is $30 \mathrm{~g}$ in this paper. Barrel roll maneuver makes the image point of the aircraft do continuous circle motion on the focal plane of the seeker. Missile is asked to change the direction of motion all the time, thus leading to lose the target because of the limit of the overload [11]. Snake maneuver is also named $S$ maneuver, which is mainly composed of vertical-S maneuver and horizontal- $S$ maneuver. These two types of maneuvers do not have essential difference [12]. Horizontal-S maneuver is simulated in detail in our paper, the theory of which is similar to barrel roll maneuver to avoid the missile.

\section{Establishment of Model}

\subsection{Model of the Aircraft}

3.1.1. Model of Aircraft Maneuver. The movement of aircraft can be learnt from solving equations of six degrees of freedom, but it is difficult to obtain coefficients of aerodynamics and operation quantities, such as rudder angle, which bring difficulties into solving the equations. To ensure the credibility of the aircraft trajectory, the actual flight data got from the recorder of flight data is adopted.

The flight data has been collected from 200 sorties of an aircraft and the maneuvers are identified [13], from which 50 maneuvers have been sampled to build up the maneuver database. One data of maneuver database is chosen, such as barrel roll maneuver. To ensure the authenticity of the aircraft trajectory, assuming that the initial time is known, the flight information (such as position, condition, and speed) of the aircraft is obtained by interpolating in the simulation.

3.1.2. Model of Aircraft Radiation. Aircraft radiations are composed of aircraft plume radiation, aircraft hot parts radiation, skin radiation, reflected sunshine radiation, reflected earthshine radiation, and reflected skyshine radiation [14]. Aircraft plume radiation and aircraft hot parts radiation are collectively called exhaust system radiation and the reflected radiation is so weak that can be ignored [15], so we just analyse skin radiation and exhaust system radiation in this paper [16].

Calculation of the aircraft exhaust system radiation model by theoretical formula is not only complicated but also has a gap compared with real data. So the measured radiation intensity of the exhaust system at different speed and different height is adopted in this paper. The relationship between sight angle and radiation intensity of the exhaust system is stored in database.

Supposing that $I_{s}$ is radiation intensity of skin, $I_{n}$ is the measured radiation intensity of exhaust system. Firstly, the coordinate systems are established. Ground coordinate system fixed on the ground is inertial coordinate system, whose original point $O$ is the projection of the coordinate onto the horizontal plane at the time of foils deployment. $\mathrm{O} y_{g}$ axis is vertical upward. $\mathrm{O} x_{g}$ and $\mathrm{O} z_{g}$ axes are in the horizontal plane and constitute right-handed coordinate system with $O y_{g}$ axis. Body coordinate system's original point $O_{b}$ is the aircraft centroid. $O x_{b}$ axis is the vertical axis of aircraft and forward is positive. $\mathrm{O} y_{b}$ axis is in the symmetry plane of aircraft and upward is positive. $O z_{b}$ axis constitutes right-handed coordinate system with $O x_{b}$ and $O y_{b}$ axes. The relationship between coordinate systems is shown in Figure 1.

Secondly, the angle $\gamma$ between line-of-sight and $x_{b}$ axis is calculated. Suppose that the aircraft coordinate is $x_{0}, y_{0}, z_{0}$ and the missile coordinate is $x_{1}, y_{1}, z_{1}$ in ground coordinate system. The transition matrix from ground coordinate system to body coordinate system is $\mathbf{L}_{\mathbf{g}}^{\mathbf{b}}$ :

$$
\left[\begin{array}{c}
x_{b} \\
y_{b} \\
z_{b}
\end{array}\right]=\mathbf{L}_{g}^{b}\left[\begin{array}{c}
x_{1}-x_{0} \\
y_{1}-y_{0} \\
z_{1}-z_{0}
\end{array}\right]
$$




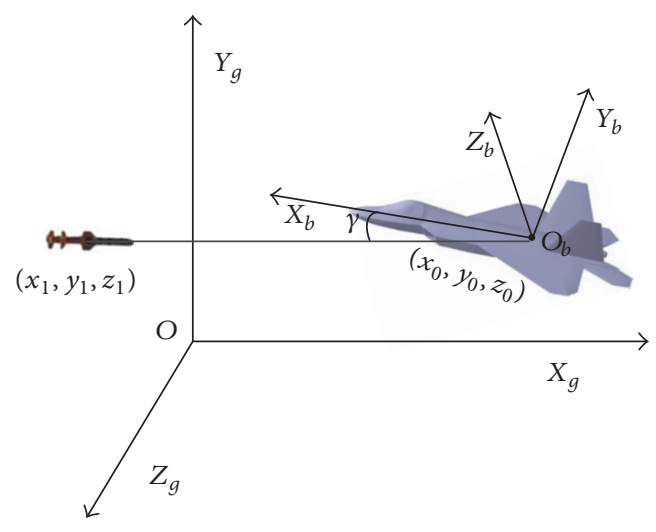

FIGURE 1: The relationship of each coordinate system.

so $\gamma$ is

$$
\gamma=\arccos \left(\frac{x_{b}}{\sqrt{x_{b}^{2}+y_{b}^{2}+z_{b}^{2}}}\right) .
$$

The radiation intensity of exhaust system $I_{n}$ in the direction of line-of-sight can be solved by interpolating and inquiring about the relationship between radiation intensity of exhaust system and sight angle within database.

Thirdly, the radiation intensity of aircraft skin $I_{s}$ is calculated:

$$
I_{s}=\frac{1}{\pi} \int_{\lambda_{1}}^{\lambda_{2}} \varepsilon \frac{c_{1}}{\lambda^{5}} \frac{1}{e^{c_{2} / \lambda T_{s}}-1} d \lambda \sum_{k=1}^{N} A_{k} \cos \theta_{k},
$$

where $I_{s}$ is the radiation intensity of skin in the wave range from $\lambda_{1}$ to $\lambda_{2}, \varepsilon$ is the emissivity of the skin, $\theta_{k}$ is included angle between sight direction and outer normal direction of skin surface element, and $A_{k}$ is skin surface element. $T_{b}$ is the stagnation temperature, which is mainly related to atmosphere temperature and flight velocity. The average temperature in the surface of aircraft skin is $T_{s}$, which is approximately $k$ times higher than $T_{b}$; that is,

$$
T_{s}=k T_{b} .
$$

The selection of $k$ should consider aerodynamic heating, environment radiation (solar radiation and atmosphere radiation), and internal heating (engine, jet nozzle, and electronic cabin). It will be confirmed by comprehensive action of thermal convection, heat conduction, and thermal radiation [17]. When the surface of aircraft is smooth (without external weapons) and does not expose to the sunshine and the effect of heat insulation is well, the value of $k$ is little. The range of $k$ is from 0.7 to 0.95 .

Lastly, it is concluded that

$$
I=I_{s}+m I_{n}
$$

where $I$ is the total radiation intensity and $m$ is the exhaust system radiation correction factor of taking the skin covering into consideration.
3.2. Model of Surface-Type IR Decoy. Surface-type IR decoy consists of many foils, which is suppressed in the sealed barrel and isolated from air. Once exposed in the air, the foils self-ignite rapidly. The continuous burning time of the foils is about two or three seconds (sec) with a temperature maintained in certain range, which changes slowly compared with point-type IR decoy. At the same time the temperature will not be too high, so the radiation characteristics are more like the aircraft [18].

Surface-type IR decoy is one of the most effective IR interference equipment, which can produce large area IR radiation and shield the image of target IR radiation. It will seriously interfere with the distinguishment of the seeker. Surface-type IR decoy drops sharply by gravity and aerodynamic drag after deploying, whose trajectory is similar to parabola.

3.2.1. Motion Model of Surface-Type IR Decoy. With the influence of gravity, aerodynamic force, internal force, random factors, and so on, the motion laws of foils are different and complex; therefore, we regard the surface-type IR decoy as a whole to study the shape of spread and the motion trajectory of centroid instead of studying the motion law of every foil.

(1) Motion Equation of Centroid. The kinematics equations in ground coordinate system are shown as follows:

$$
\begin{aligned}
\frac{d v_{D x}(t)}{d t} & =a_{r}(t) \frac{v_{D x}(t)}{v_{D}(t)}, \\
\frac{d v_{D y}(t)}{d t} & =a_{r}(t) \frac{v_{D y}(t)}{v_{D}(t)}-g, \\
\frac{d v_{D z}(t)}{d t} & =a_{r}(t) \frac{v_{D z}(t)}{v_{D}(t)}, \\
v_{D}(t) & =\sqrt{\left(v_{D x}(t)\right)^{2}+\left(v_{D y}(t)\right)^{2}+\left(v_{D z}(t)\right)^{2}} .
\end{aligned}
$$

It can be known from Bernoulli equation that

$$
a_{r}(t)=-\frac{0.5 C_{d} S \rho v_{D}(t)^{2}}{m}
$$

where $v_{D}(t)$ is the velocity of decoy centroid at time $t . v_{D x}(t)$, $v_{D y}(t)$, and $v_{D z}(t)$ is the component velocity of $v_{D}(t)$ in the axes of $X_{g}, Y_{g}$, and $Z_{g} . C_{d}$ is the drag coefficient. $S$ is the frontal area of the spread surface-type IR decoy. $\rho$ is the air density. $a_{r}(t)$ is the acceleration generated by aerodynamic drag.

The decoy velocity is much less than the local speed of sound. Assume that $C_{d} S$ is invariant in the movement of decoy. During the flight of decoy, when the drag and gravity is equal and opposite, $v_{D}(t)$ is invariant and the decoy descends at an even speed:

$$
0.5 C_{d} S \rho v_{D}(t)^{2}-m g=0,
$$

where $C_{d} S$ is as follows:

$$
C_{d} S=\frac{2 m g}{\left(\rho v_{D}^{2}\right)}
$$




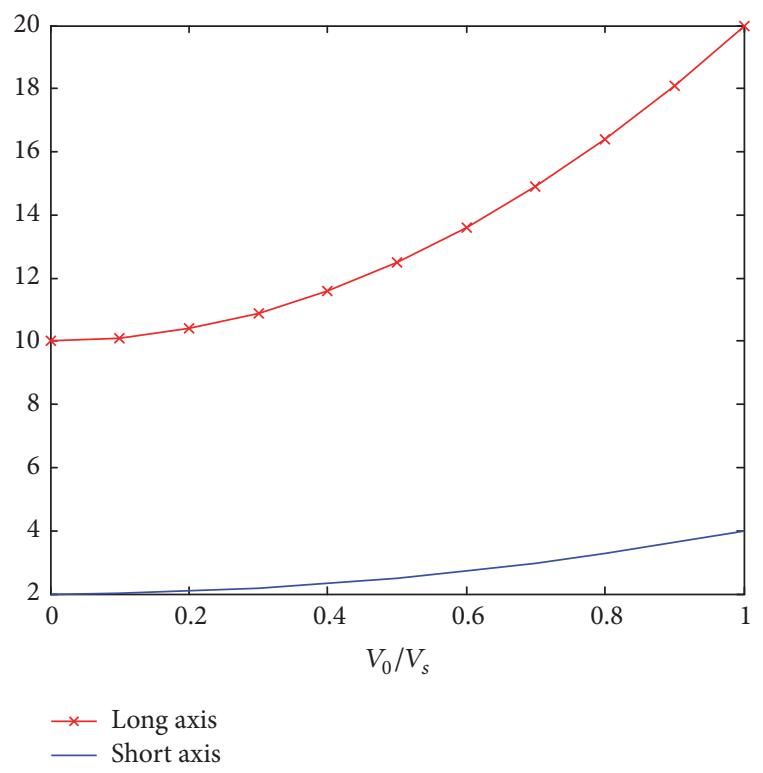

FIGURE 2: The long and short axis curve of surface-type IR decoy spreading shape.

where $v_{D}$ is the uniform decline speed which can be obtained by surface-type IR decoy low altitude deploying experiment.

The decoy centroid coordinate at time $t$ is as follows:

$$
\begin{aligned}
& x(t)=\int_{t_{o}}^{t} v_{D x}(t) d t+x_{o}, \\
& y(t)=\int_{t_{o}}^{t} v_{D y}(t) d t+y_{o}, \\
& z(t)=\int_{t_{o}}^{t} v_{D z}(t) d t+z_{o} .
\end{aligned}
$$

(2) Spread Shape Equations of Decoy. Surface-type IR decoy spreading shape is approximate to ellipsoid and the direction of long axis is similar to the decoy centroid motion direction. The variation of long axis ranges from ten to twenty meters, and the short is three to five meters [19], while the values of long and short axis have relation with velocity. The relationship between the length of long and short axis and the initial speed of decoy is shown in Figure 2.

Fitting the curve in Figure 2, the approximate fitting equations are shown as follows:

$$
\begin{aligned}
& r_{a}=10\left(1+\left(\frac{v_{0}}{v_{s}}\right)^{2}\right), \\
& r_{b}=2\left(1+\left(\frac{v_{0}}{v_{s}}\right)^{2}\right),
\end{aligned}
$$

where $r_{a}$ and $r_{b}$ are the long axis and short axis, respectively, $v_{s}$ is the local speed of sound, $v_{0}<v_{s}$, and $v_{0}$ is the initial speed of the decoy.

3.2.2. Radiation Model of Surface-Type IR Decoy. The radiation characteristics of surface-type IR decoy are decided by

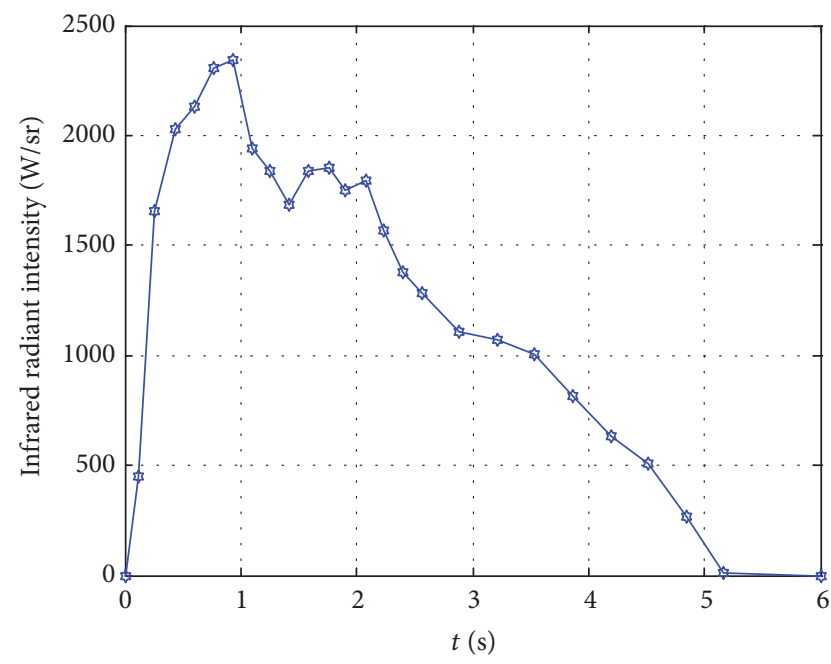

FIGURE 3: Radiation intensity variation curve of surface-type IR decoy.

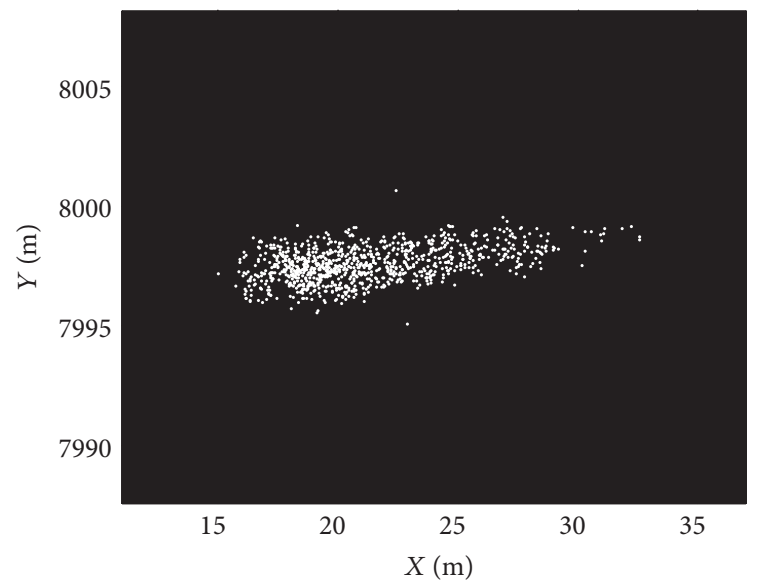

FIGURE 4: Side view of the surface-type IR decoy gray level distribution.

the radiation characteristics of the foils diffusing in the air. The foils are adhered to Magnesium and Teflon [20], which will generate much heat rapidly when exposed to the air [21]. The sampling test is done to measure the variation of foils' diffusing temperature in the whole deployment process. And then draw the curve of foils' temperature varying with time after interpolation and smooth processing. The medium wave band radiation intensity of the surface-type IR decoy ranges from $1000 \mathrm{~W} / \mathrm{sr}$ to $2500 \mathrm{~W} / \mathrm{sr}$. Under the condition of $8000 \mathrm{~m}$ height and $1 \mathrm{Ma}$ speed, the interpolation curve of the dynamic radiation intensity of the surface-type IR decoy is shown in Figure 3 and the gray level distribution of the surface-type IR decoy is shown in Figures 4 and 5.

\section{Simulation Result}

\subsection{Maneuver Simulation}

4.1.1. Barrel Roll Maneuver Simulation. Supposing that the target aircraft and the attacker are all in $9200 \mathrm{~m}$ height 


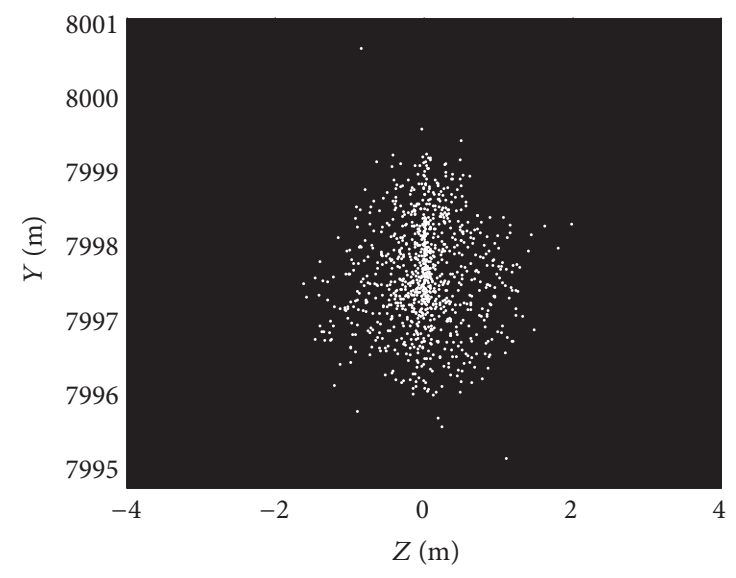

FIGURE 5: Front view of the surface-type IR decoy gray level distribution.

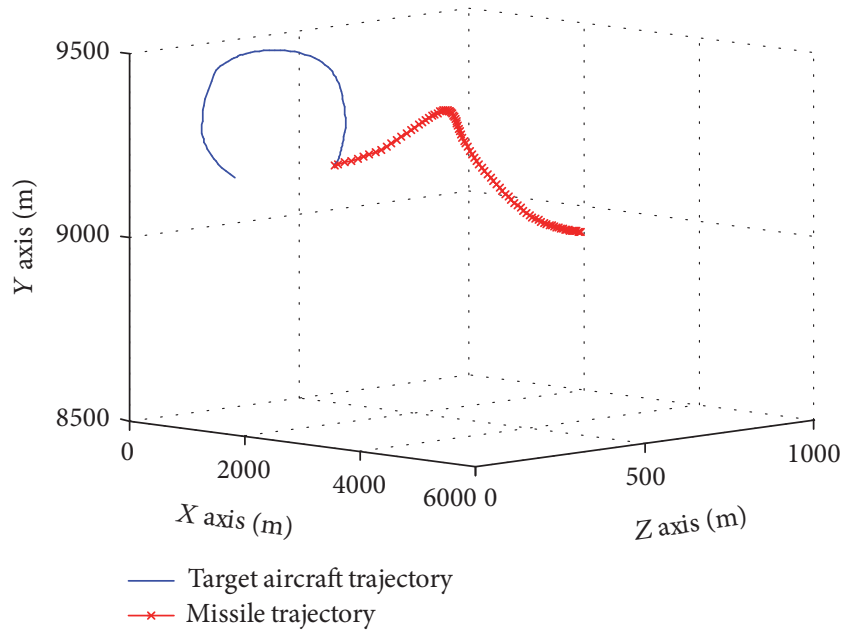

FIGURE 6: Simulation trajectory of target aircraft performing $200 \mathrm{~m}$ barrel roll radius of barrel roll maneuver.

and fly in opposite direction, the target aircraft flies along the positive $X$ axis at the speed of $200 \mathrm{~m} / \mathrm{s}$. The attacker launches the missile at the distance of $6000 \mathrm{~m}$ and the target aircraft performs barrel roll maneuver at the same time. The simulation results are shown in Figures 6-9. The barrel roll radius is the radius of the cylindrical curved surface expanding generated by the spiral trajectory of barrel roll maneuver which is not the real radius of motion trajectory.

4.1.2. Horizontal-S Maneuver Simulation. Suppose that the target aircraft and the attacker are all in $8000 \mathrm{~m}$ height and they fly in opposite direction at the speed of $200 \mathrm{~m} / \mathrm{s}$. The attacker launches the missile at the distance of $6000 \mathrm{~m}$ and the target aircraft performs horizontal-S maneuver at the same time [22]. The simulation results are shown in Figures 10 and 11.

Keeping initial conditions invariant and repeating both of maneuvers 2000 times, the hitting results are as shown in Table 1.

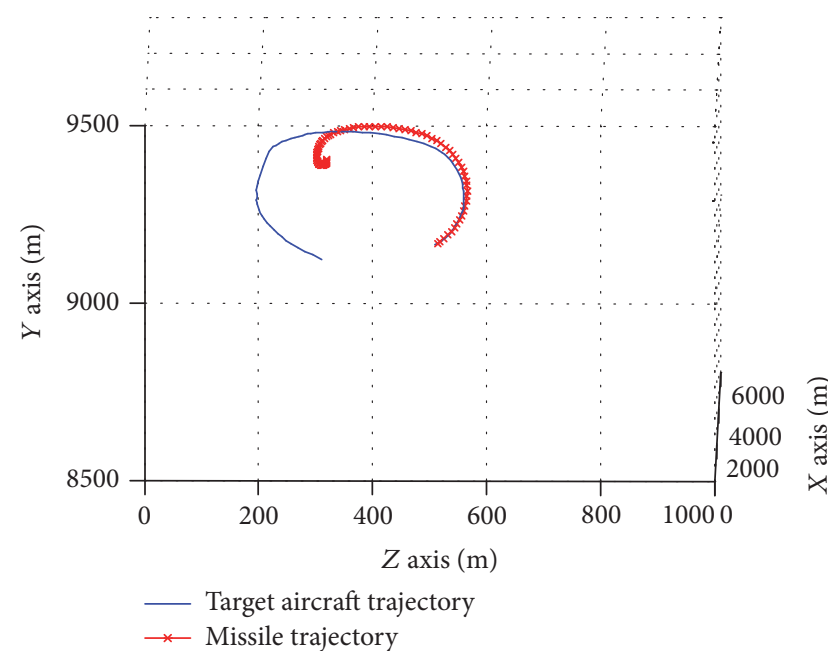

FIGURE 7: Simulation trajectory of the negative $X$ axis direction.

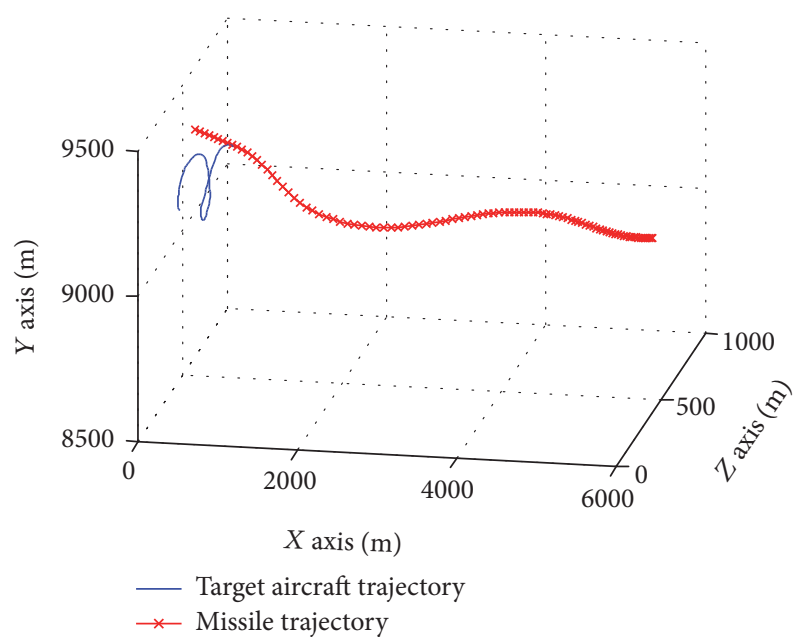

FIGURE 8: Simulation trajectory of target aircraft performing $125 \mathrm{~m}$ barrel roll radius of barrel roll maneuver.

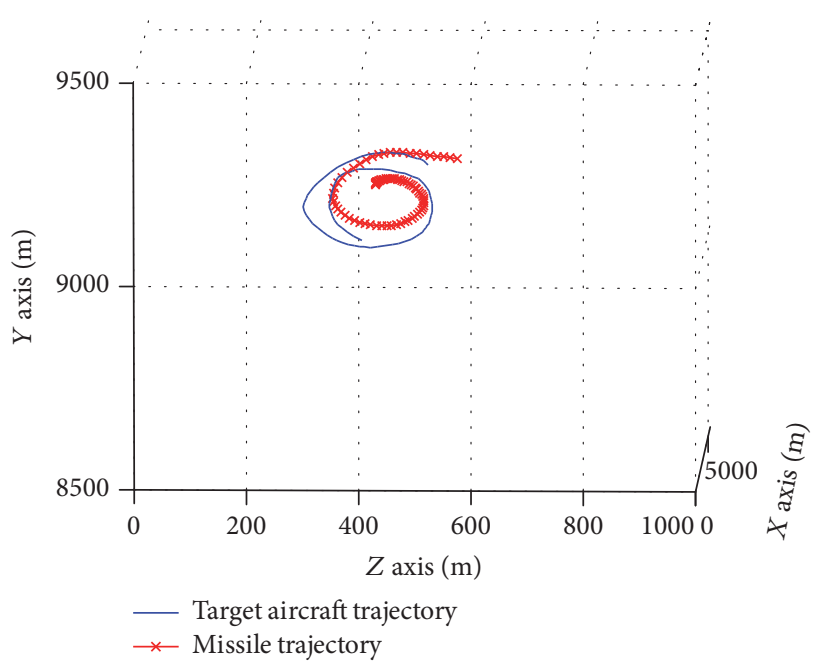

FIGURE 9: Simulation trajectory of the negative $X$ axis direction. 


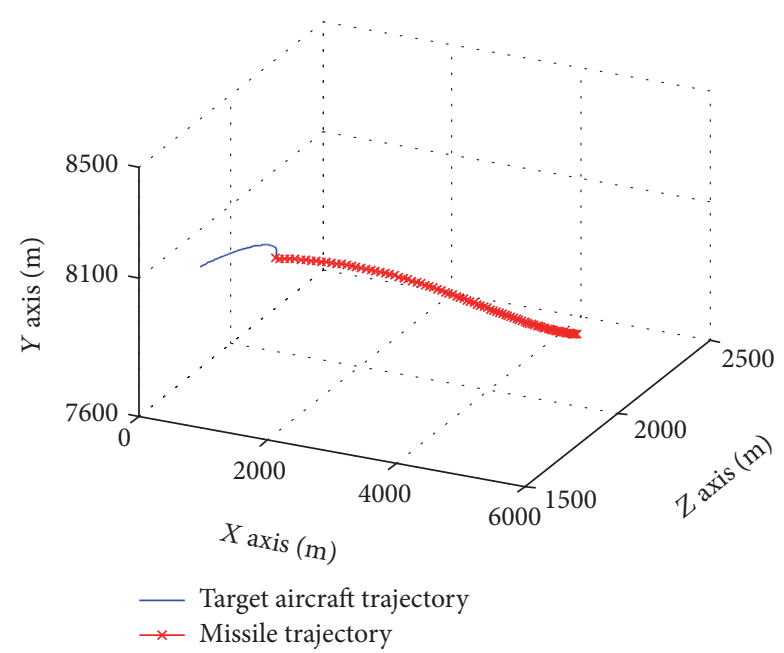

FIGURE 10: Simulation trajectory of target aircraft performing $1000 \mathrm{~m}$ radius of horizontal- $S$ maneuver.

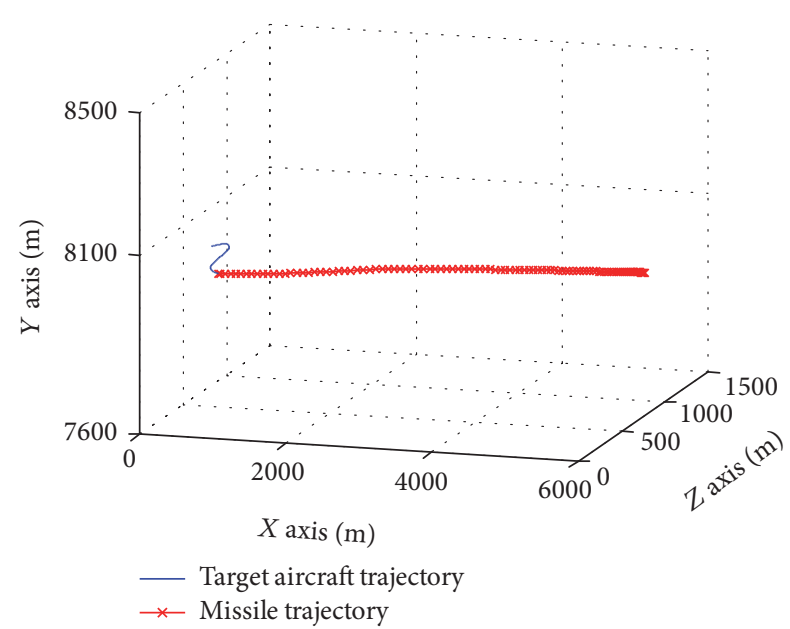

FIGURE 11: Simulation trajectory of target aircraft performing $500 \mathrm{~m}$ radius of horizontal- $S$ maneuver.

TABLE 1: Comparison of different maneuvers to the missile hit rate.

\begin{tabular}{lcccc}
\hline & \multicolumn{2}{c}{$\begin{array}{c}\text { Horizontal-S } \\
\text { maneuver }\end{array}$} & \multicolumn{2}{c}{$\begin{array}{c}\text { Barrel roll } \\
\text { maneuver }\end{array}$} \\
\hline Maneuver radius/m & 1000 & 500 & 200 & 125 \\
Hit rate/\% & 98.4 & 91.8 & 85.4 & 73.5 \\
\hline
\end{tabular}

It is concluded from Table 1 that when the coming missile is from the frontage of the target aircraft, it prefers to perform barrel roll maneuver rather than snake maneuver to avoid missile.

4.2. Airborne IR Jamming Simulation. It is concluded from maneuver simulation that barrel roll maneuver with small radius and large overload is more beneficial for aircraft to avoid missile. The airborne IR jamming simulation is that the target performs small radius barrel roll maneuver and small radius snake maneuver, timely deploying surface-type IR decoy to interfere missile in this chapter.

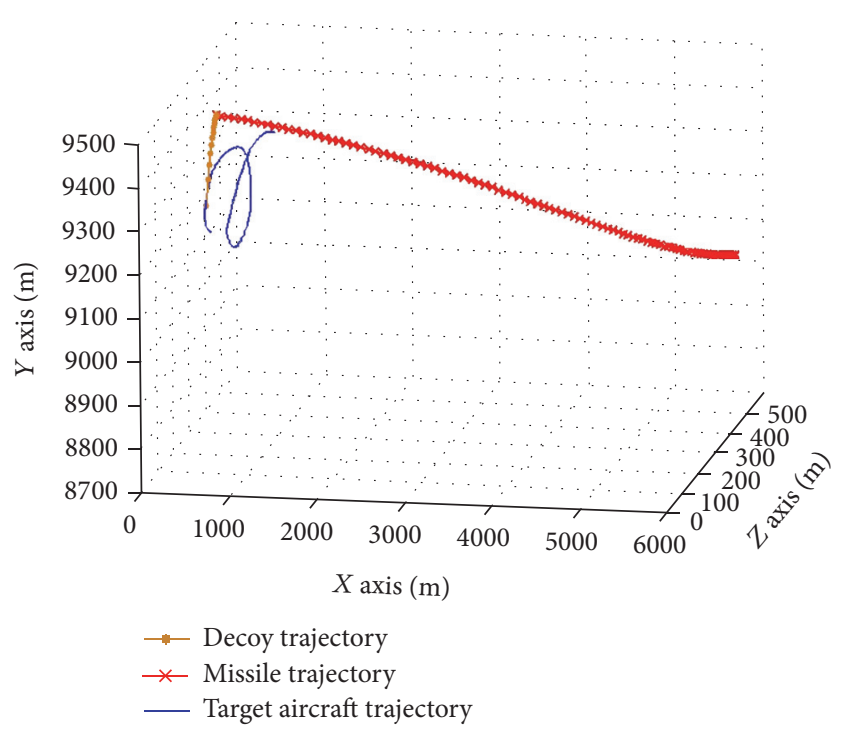

FIGURE 12: Simulation trajectory combined barrel roll maneuver with surface-type IR decoy.

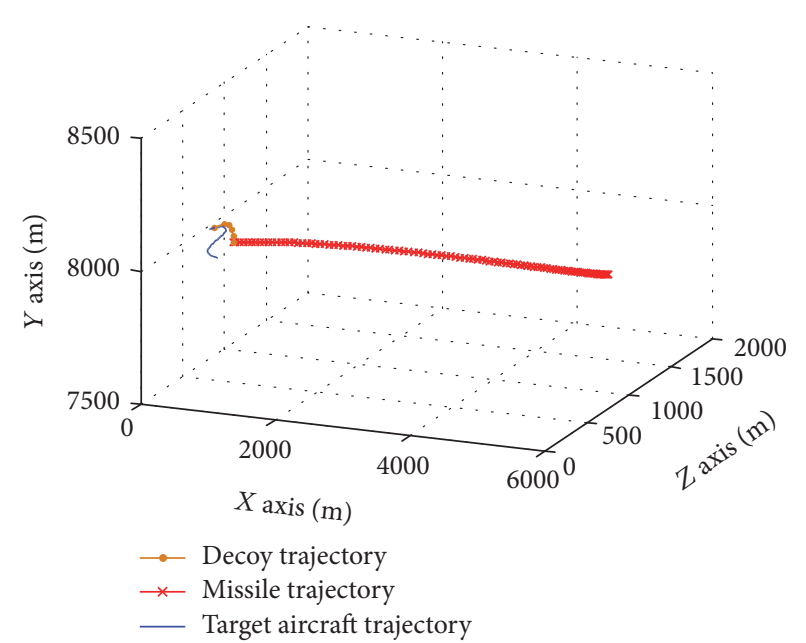

FIGURE 13: Simulation trajectory combined horizontal-S maneuver with surface-type IR decoy.

Assume that the target aircraft performs barrel roll maneuver of $125 \mathrm{~m}$ barrel roll radius and horizontal- $S$ maneuver of $500 \mathrm{~m}$ radius, respectively, and missile launch time is zero hours, and surface-type IR decoy is deployed after $0.5 \mathrm{sec}$; meanwhile other conditions are similar to maneuver simulation. The simulation results are shown in Figures 12 and 13.

The target aircraft deploys one and four surface-type IR decoys (time interval is $0.1 \mathrm{sec}$ ), respectively, when receiving the airborne alarming signal and repeats both simulations 2000 times. The simulation results are as shown in Table 2.

\section{Simulation Analysis}

5.1. Maneuver Simulation Analysis. It is concluded from maneuver simulation that when the target aircraft performs barrel roll maneuver to avoid missile, the trajectory of missile 
TABLE 2: Comparison of different number of surface-type IR decoys to missile hit rate.

\begin{tabular}{lcc}
\hline & $\begin{array}{c}\text { Barrel roll } \\
\text { maneuver }\end{array}$ & $\begin{array}{c}\text { Horizontal-S } \\
\text { maneuver }\end{array}$ \\
\hline $\begin{array}{l}\text { Maneuver radius/m } \\
\text { One surface-type IR decoy hit } \\
\text { rate/\% }\end{array}$ & 125 & 500 \\
$\begin{array}{l}\text { Four surface-type IR decoys } \\
\text { hit rate/\% }\end{array}$ & 50.5 & 88.4 \\
\hline
\end{tabular}

is approximately close to the shape of barrel roll. It will make the missile fly with big overload all the time. When the target is flying opposite to missile, despite the fact that missile consumes much energy because of the big overloaded barrel roll maneuver, the remaining fuels are enough for hitting the target. Therefore, the target aircraft can avoid the missile only by the limit of maximum overload of the missile to make it disappear in the missile field of view.

When the target aircraft performs barrel roll maneuver of $200 \mathrm{~m}$ barrel roll radius (the real trajectory radius is about $800 \mathrm{~m}$ ), the missile hits the target. When the target aircraft performs barrel roll maneuver of $125 \mathrm{~m}$ barrel roll radius (the real trajectory radius is about $500 \mathrm{~m}$ ), the missile misses the target.

When the target aircraft performs small radius barrel roll maneuver, the missile is asked to reduce the radius to fly to target, which will make the missile fly with big overload. However, the radius of missile is larger than the target. When the distance between the target and missile is short, the missile is supposed to reduce the radius of barrel roll at the right time to hit the target [23]. The computed overload calculated by the missile computer is likely to exceed the maximum available overload, thus resulting in the miss of the target.

But for horizontal-S maneuver, the probability of avoiding missile for the target aircraft performing horizontal- $S$ maneuver of $500 \mathrm{~m}$ radius is higher than the $1000 \mathrm{~m}$ radius. However the radius is, the effect of avoiding missile is not good, as the hitting rate is still high.

When the target aircraft performs horizontal-S maneuver to avoid missile, the trajectory of missile is approximately close to the shape of horizontal-S maneuver when tracking the target at the same time. Compared with barrel roll maneuver, the overload of horizontal-S maneuver is only along the horizontal direction, which means that the maximum radius of horizontal direction is smaller. When the distance between the target and missile is short, the missile only needs to make a sharp turn in the horizontal direction and then quickly point to the target and hit the target.

It is concluded from simulation that as far as the effect of avoiding missile is concerned, the horizontal- $S$ maneuver of $500 \mathrm{~m}$ radius is similar to the barrel roll maneuver of $200 \mathrm{~m}$ radius. All in all, the smaller the snake maneuver radius is, the more successful of avoiding the missile. Consider the fact that the maximum overload that the pilot can bear in a short time is about $10 \mathrm{~g}$, what limits the minimum turning radius.
The smaller radius of barrel roll maneuver is propitious to avoid missile, of which the barrel roll maneuver of $125 \mathrm{~m}$ radius is the most effective.

5.2. Airborne IR Jamming Simulation Analysis. It is concluded from maneuver simulation that the effect of defense is best when the target aircraft performs barrel roll maneuver of small radius ( $125 \mathrm{~m}$ to $150 \mathrm{~m}$ ), meanwhile deploying multiple surface-type IR decoys [24].

The initial deployment speed of surface-type IR decoy is much slower compared with the speed of the target aircraft, so surface-type IR decoy flies approximately along the tangent line of the maneuver trajectory and the velocity decreases sharply. When the target aircraft deploys surface-type IR decoy, the missile gets into antijamming state and remembers the radiation characteristics of the target image before antijamming state. When surface-type IR decoy separates from the target aircraft, owing to the slow speed of surface-type IR decoy, the separation position of surface-type IR decoy in the focal plane of the seeker is more close to the position of the target image before antijamming state. At the same time the missile exits the antijamming state.

Firstly, the seeker chooses the target which is almost similar to the radiation characteristics of the target image in the memory. The radiation characteristics of surface-type IR decoy are similar to the target aircraft, which do interference to the missile. Moreover, the missile judges from distance and selects the point which is the closest to the true target in the memory before entering into antijamming state to track. Because the speed of surface-type IR decoy decreases sharply, the position is near to the position in the memory of missile. Therefore, the target aircraft which performs small radius barrel roll maneuver accompanied with the decoy is more likely to avoid the missile.

The spread shape of surface-type IR decoy is about ellipse, whose outline is different from the aircraft, while the radiation intensity of surface-type IR decoy is larger than the aircraft. Therefore, the missile can distinguish aircraft from surface-type IR decoy based on outline and radiation intensity of the memorial target image. And the effect of deploying just one decoy is not obvious. However, when deploying four decoys, some decoys are still in the state of launching and the IR image coincides with the aircraft when the missile gets out antijamming state and the missile will take the radiation characteristics of coincided IR image into memory. When the IR image of surface-type IR decoy is separated from the target aircraft, the image characteristics of both are close to the image of the memorial target of the missile and it will jam missile seriously. The missile is likely to track the surface-type IR decoy and miss the target [25].

So the best defensive strategy for the target aircraft is performing small radius barrel roll maneuver accompanied with deploying two to four decoys with the deployment time interval of $0.1 \mathrm{sec}$. The earlier the deployment time is, the better the jamming effect is. Meanwhile the pilot should observe the trajectory of missile all the time [26]. Assuming that the missile tracks the decoys instead of the target aircraft after deploying decoys, the target aircraft should exit barrel roll maneuver and fly away from the sight direction of missile. 
Under the condition that the missile still tracks the target aircraft, the target should deploy the decoys once again, of which the way is similar to the first time. Meanwhile the pilot should observe the trajectory of missile again.

\section{Conclusions}

Firstly, the models of aircraft and surface-type IR decoy based on the realistic data of flight and exhaust system radiation are established, which have high reliability. Secondly, the most effective maneuver of avoiding missile is simulated, when the coming missile is from the front of the target aircraft. Finally, on the basis of the most effective maneuver, the aircraft deploys surface-type IR decoys at the same time, looking for the best strategy of defense.

The defense strategy acquired by simulation is of high reliability and fits for the real air combat, which is propitious to improve the survivability of aircraft. The main conclusions can be drawn as follows:

(1) When the missile comes from the front of aircraft, the best strategy of defense is performing small radius barrel roll maneuver, meanwhile deploying multidecoys.

(2) When receiving the alarm signal from the alarm system, the pilot should deploy surface-type IR decoy rapidly and multiple decoys are better than one. The time interval of decoy should not be large and $0.1 \mathrm{sec}$ is okay.

(3) Observe the direction of the coming missile when the pilot is performing maneuver. After deploying one set of decoys, if the missile tracks the decoys, the target aircraft exits barrel roll maneuver and flies away from the sight direction of missile. On the other side the target aircraft should deploy another set of decoys once again and the time interval should be similar to the first time and meanwhile the pilot should observe the trajectory of missile again until the interfering is successful.

But the establishment of decoy model is relatively simple which has some gap with reality and it does not analyse the jamming effect of multidecoys with different deploying time and deploying interval time to missile. These will be studied in further research.

\section{Competing Interests}

The authors declare no potential conflict of interests with respect to the research, authorship, and/or publication of this article.

\section{Acknowledgments}

This manuscript is supported by the Natural Science Foundation of China (no. 61471390).

\section{References}

[1] T.-W. Bae, B.-I. Kim, Y.-C. Kim, and S.-H. Ahn, "Jamming effect analysis of infrared reticle seeker for directed infrared countermeasures," Infrared Physics \& Technology, vol. 55, no. 5, pp. 431-441, 2012.

[2] K. Virtanen, T. Raivio, and P. Raimo, "Modeling pilot's sequential maneuvering decisions by a multistage influence diagram," in Proceedings of the AIAA Guidance, Navigation, and Control Conference, pp. 1-11, Montreal, Canada, 2001.

[3] T. Armon, R. Janjoris, S. Pieter et al., "Dynamic scripting with team coordination in air combat simulation," in Modern Advances in Applied Intelligence: 27th International Conference on Industrial Engineering and Other Applications of Applied Intelligent Systems, IEA/AIE 2014, Kaohsiung, Taiwan, June 3-6, 2014, Proceedings, Part I, vol. 8481 of Lecture Notes in Computer Science, pp. 440-449, Springer, Berlin, Germany, 2014.

[4] V. Arthur, "Missile avoidance manoeuvres with simultaneous decoy deployment," in Proceedings of the AIAA Guidance, Navigation, and Control Conference, pp. 10-13, Toronto, Canada, 2009.

[5] F. Imado and S. Miwa, "Missile guidance algorithm against high-g barrel roll maneuvers," Journal of Guidance, Control, and Dynamics, vol. 17, no. 1, pp. 123-128, 1994.

[6] X. Xinhui, Z. Xuebin, F. Li et al., "Research on effects of fighter HGB maneuvering terminal evasion," Fire Control \& Command Control, vol. 41, pp. 97-107, 2016.

[7] L. Jianwei and W. Qiang, "Aircraft-skin infrared radiation characteristics modeling and analysis," Chinese Journal of Aeronautics, vol. 22, no. 5, pp. 493-497, 2009.

[8] J. Wimde, P. Sebastiaan, B. Vanden et al., "IR seeker simulator to evaluate IR decoy effectiveness," in Proceedings of the Targets and Backgrouds VIII: Characterization and Representation, vol. 4718 of Proceedings of SPIE, pp. 164-172, 2002.

[9] H. R. Sonawane and S. P. Mahulikar, "Effect of missile turn rate on aircraft susceptibility to infrared-guided missile," Journal of Aircraft, vol. 50, no. 2, pp. 663-666, 2013.

[10] Y. Dong and J. Ai, "Trial input method and own-aircraft state prediction in autonomous air combat," Journal of Aircraft, vol. 49, no. 3, pp. 947-954, 2012.

[11] P.-X. Wei, W.-X. Jing, and C.-S. Gao, "An analysis of maneuvering overloads in attack-defense confrontation," Journal of Astronautics, vol. 34, no. 2, pp. 179-185, 2013.

[12] F. Yuanwei, X. Xiaohua, and W. Yanzheng, "Comparison of penetration effect of two kinds of $S$ maneuver missile against anti-missile naval gun," Fire Control \& Command Control, vol. 36, pp. 107-113, 2011.

[13] Y. Wang, J. Dong, X. Liu, and L. Zhang, "Identification and standardization of maneuvers based upon operational flight data," Chinese Journal of Aeronautics, vol. 28, no. 1, pp. 133-140, 2015.

[14] S. P. Mahulikar, H. R. Sonawane, and G. A. Rao, "Infrared signature studies of aerospace vehicles," Progress in Aerospace Sciences, vol. 43, no. 7-8, pp. 218-245, 2007.

[15] M. A. Noah, J. Kristl, J. W. Schroeder, and B. P. Sandford, "NIRATAM-NATO infrared air target model," in Proceedings of the Surveillance Technologies, Proceedings of SPIE, pp. 275-282, April 1991.

[16] W. Chaozhe, T. Zhongxiang, and L. Yongzhi, "Evaluation method of stealth aircraft's IR radiation measurement," IR and Laser Engineering, vol. 41, pp. 2891-2897, 2012. 
[17] X. Xinlin, A. Qing, and R. Depeng, "Analysis on the transient temperature-fields for ir radiation of aircraft skin," Journal of Infrared, Millimeter and Terahertz Waves, vol. 26, pp. 174-177, 2007.

[18] T. Qi, L. Jianxun, F. Yangwang et al., "Simulation research on surface-type IR decoy for jamming IR imaging guided missile," IR and Laser Engineering, vol. 44, pp. 1150-1157, 2015.

[19] H. Chao, Z. Guozhi, Y. Zhiqiang, and N. Jitao, "Study on the kinematics characteristic of chaff release," Initiators \& Pyrotechnics, vol. 2, pp. 5-8, 2005.

[20] F. Xuefeng, "Study on the formula optimization of Mg/PTFE/ Viton(MTV) infrared decoy flare composition," Initiators \& Pyrotechnics, vol. 3, pp. 38-41, 2015.

[21] C. Chunsheng, D. Mengyan, L. Harfeng et al., "Research on spectrum radiation characteristics of a new type IR/ultraviolet dual color decoy," Spectroscopy and Spectral Analysis, vol. 35, pp. 1824-1829, 2015

[22] Z. Liangliang, Z. Feng, and X. Tong, "A new model of the 'Smaneuver' target and a tracking method," Electronics Optics \& Control, vol. 19, pp. 13-22, 2012.

[23] R. C. Alan, W. M. John, and H. G. Kenneth, "Trail maneuver generation and selection in the paladin tactical decision generation system," in Proceedings of the AIAA Guidance, Navigation and Control Conference, pp. 1066-1075, 1992.

[24] L. Mingshan, "Approach jamming effectiveness evaluation for surface-type IR decoy in network centric worship formation," in Proceedings of SPIE, Optical and Optoelectronic Sensing and Imaging Technology, vol. 9674, pp. 231-237, 2015.

[25] B. Guo, X. Wang, X. Wang, H. Yuan, and H. Yang, "Analysis of imaging features and tracking performance of seeker after decoys jamming," Hongwai yu Jiguang Gongcheng/Infrared and Laser Engineering, vol. 44, no. 7, pp. 2027-2033, 2015.

[26] S. Akbari and M. B. Menhaj, "New framework of a decision support system for air to air combat tasks," in Proceedings of the IEEE International Conference on Systems, Man and Cybernetics, pp. 2019-2022, October 2000. 


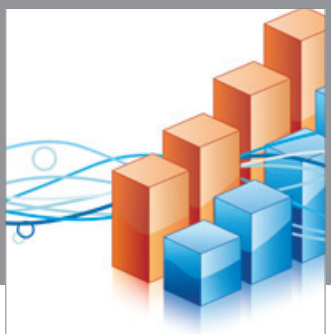

Advances in

Operations Research

vatem alat4

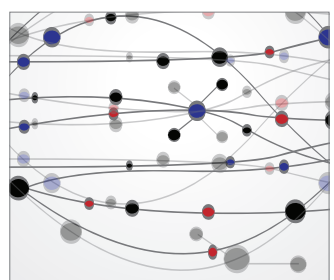

\section{The Scientific} World Journal
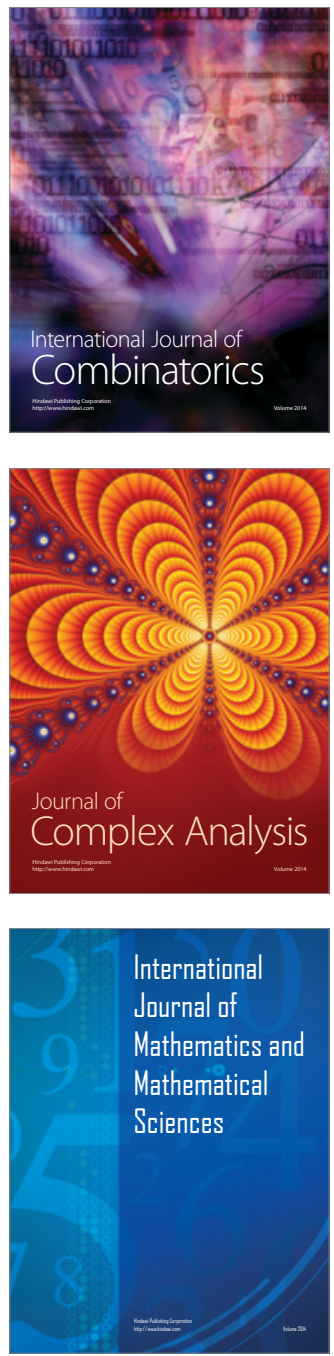
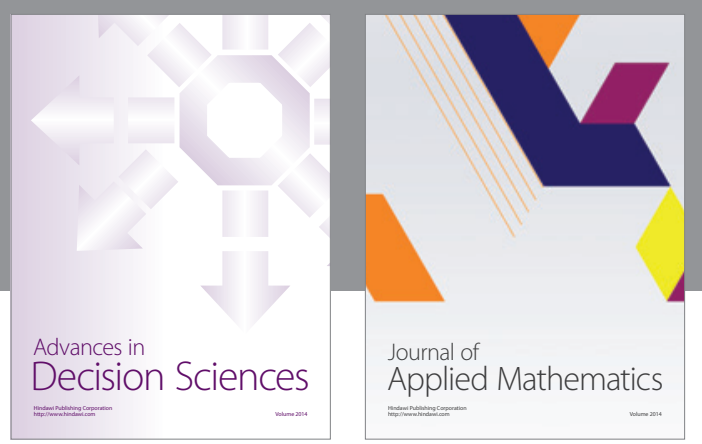

Algebra

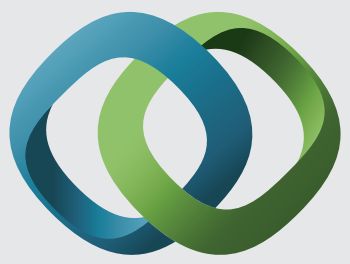

\section{Hindawi}

Submit your manuscripts at

https://www.hindawi.com
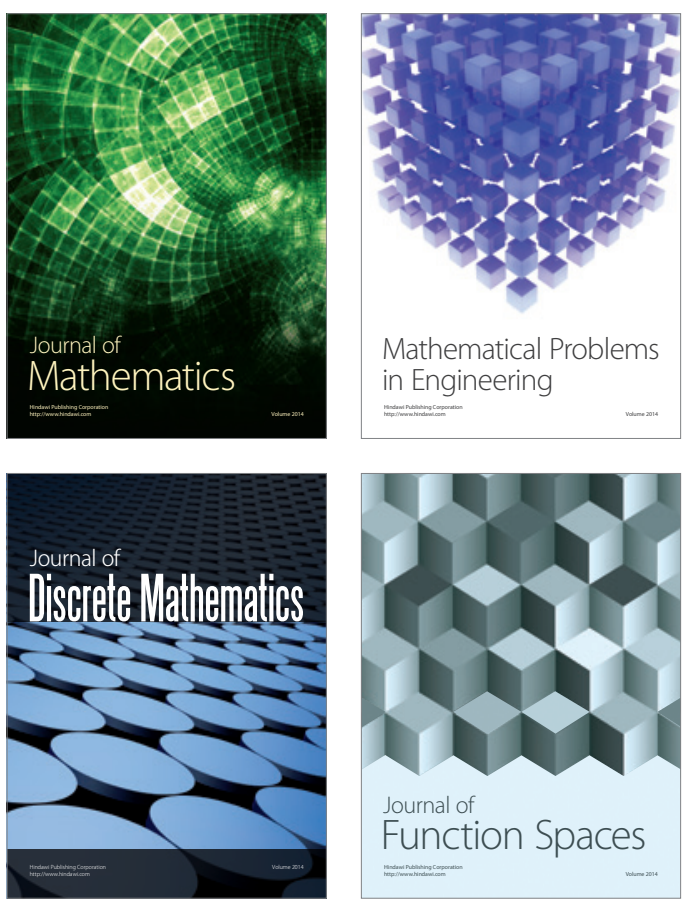

Mathematical Problems in Engineering
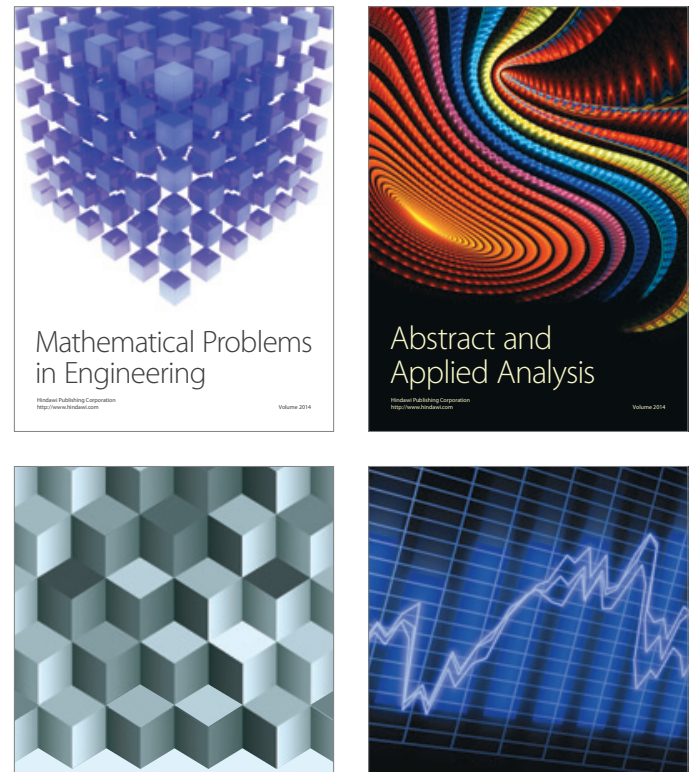

Journal of

Function Spaces

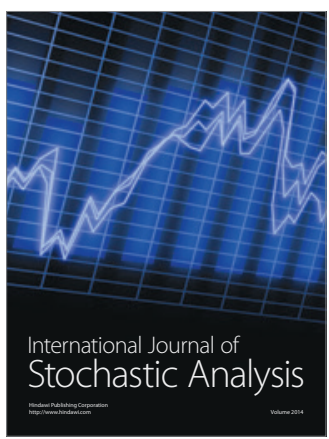

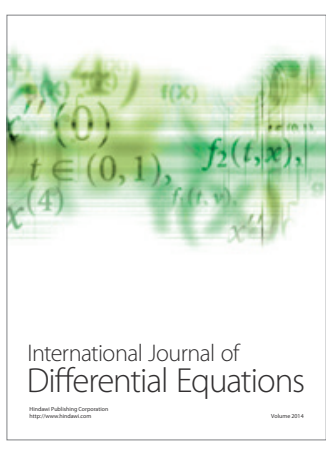
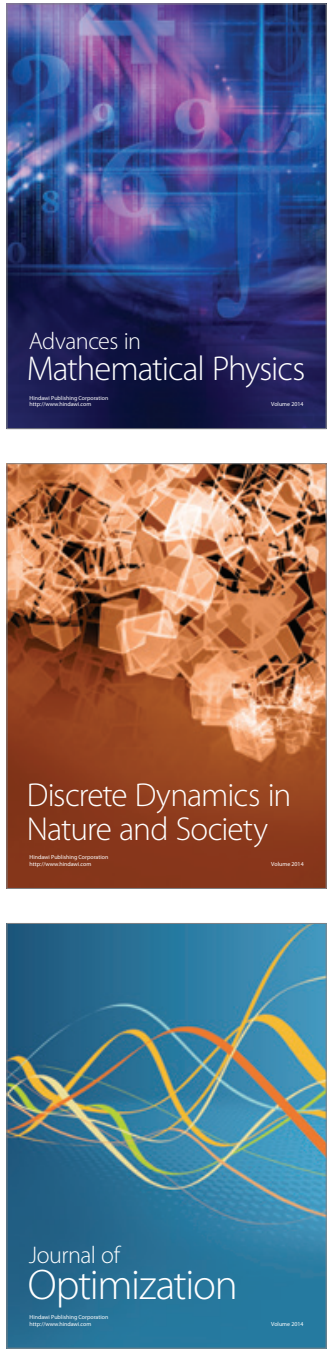